\title{
QUELQUES DESCRIPTIONS DE DÉSINGULARISATIONS PLONGÉES DE SURFACES
}

\author{
By GÉRARd GonZALEZ-SPRINBERG
}

\section{Introduction}

Soit $(S, O)$ un représentant d'un germe de surface complexe, où $O$ est un point singulier isolé. Une désingularisation de $S$ est un morphisme propre $\sigma: \hat{S} \rightarrow S$, où $\hat{S}$ est une surface lisse, $\hat{S} \backslash \sigma^{-1}(O)$ est dense dans $\hat{S}$, tel que $\sigma$ soit un isomorphisme de $\hat{S} \backslash \sigma^{-1}(O)$ sur $S \backslash\{O\}$. Etant donné un plongement de $S$ dans une variété lisse $V$, on appelle désingularisation plongée de $S \hookrightarrow V$, un morphisme propre $\sigma: \hat{V} \rightarrow V$, où $\hat{V}$ est une variété lisse, $\hat{V} \backslash \sigma^{-1}(O)$ est dense dans $\hat{V}, \sigma$ est un isomorphisme de $\hat{V} \backslash \sigma^{-1}(O)$ sur $V \backslash\{O\}$, et tel que $\sigma$ induise une désingularisation de $S$ avec des croisements normaux, Plus précisément $\sigma$ satisfait :

i) Soit $\hat{S}=\overline{\sigma^{-1}(S \backslash\{O\})}$ le transformé strict de $S$, i.e. l'adhérence de $\sigma^{-1}(S \backslash\{O\})$ dans $\hat{V}$. La restriction $\sigma_{1 \hat{S}}$ de $\sigma$ à $\hat{S}$ est une désingularisation de $S$.

ii) $\sigma^{-1}(O)$ est un diviseur de $\hat{V}$, réunion d'hypersurfaces lisses irréductibles $D_{\imath}, 1 \leqq i \leqq r$; et la famille $\left\{\widehat{S}, D_{1}, \cdots, D_{r}\right\}$ est à croisements normaux.

La condition de croisement normal sur la famille $\left\{\hat{S}, D_{1}, \cdots, D_{r}\right\}$ veut dire que, pour chaque $x \in \sigma^{-1}(O)$, les fonctions qui apparaissent dans les équations locales des variétés de la famille qui contiennent $x$ forment un système local de coordonnées ou peuvent être complétées pour former un tel système. Autrement dit, pour chaque $x \in \sigma^{-1}(O)$ il existe un système local de coordonnées de $\hat{V}$ en $x$ tel que les variétés de la famille $\left\{\hat{S}, D_{1}, \cdots, D_{r}\right\}$ qui contiennent $x$ sont des sous-espaces linéaires de coordonnées, en position générale. On a donc $\sigma^{-1}(S)$ $=\hat{S} \cup D_{1} \cup \cdots \cup D_{r}$, et le diagramme suivant est commutatif :

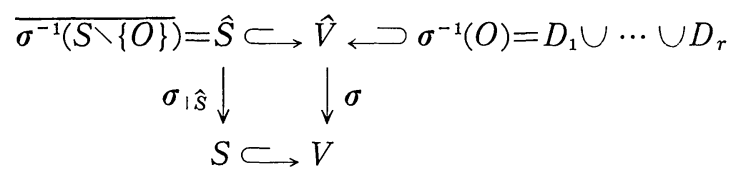

Le diviseur $\sigma^{-1}(O)$ de $\hat{V}$, dont la décomposition en composantes irréductibles est $D_{1} \cup \cdots \cup D_{r}$, est appelé le diviseur exceptionnel de $\sigma$.

Mots-Clés: Surfaces, Singularités.

Classification A.M.S.: 14B05, 14E15, 32C45.

Received October 3, 1990. 


\section{Remarques.-}

1) Pour chaque $x \in \sigma^{-1}(O) \cap \hat{S}$, la condition de croisement normal implique qu'il y a au plus deux composantes irréductibles de $\sigma^{-1}(O)$ qui contiennent $x$, car $\hat{S}$ est une variété de dimension deux.

2) La désingularisation de $S$ induite par une telle désingularisation plongée possède les propriétés de ce qu'on appelle une bonne désingularisation de $S$ : les composantes irréductibles de la fibre exceptionnelle $\sigma_{i S}^{-1}(O)$ sont lisses et à croisements normaux dans $\hat{S}$. En effet, on a $\sigma_{1 \hat{S}}^{-1}(O)=\hat{S} \cap \sigma^{-1}(O)$, et la famille des composantes irréductibles de cette intersection est à croisements normaux dans $\hat{S}$, d'où en particulier leur lissité.

L'existence de désingularisations plongées a été démontrée par $\mathrm{H}$. Hironaka, dans un cadre plus général que celui des surfaces $[\mathrm{A}-\mathrm{H}-\mathrm{V}]$. La réalisation effective et la description de telles désingularisations posent des questions non entièrement comprises.

Par exemple, si $S$ est plongée dans son espace tangent de Zariski, la géométrie intrinsèque de $S$ doit jouer un rôle dans la désingularisation plongée. Peut-on définir une notion de minimalité ou de désingularisation plongée canonique? Pour les surfaces il existe une désingularisation minimale, conséquence de la caractérisation des diviseurs de première espèce (lemme de Castelnuovo). Comment obtenir une désingularisation plongée à partir d'une désingularisation minimale? En ayant obtenu une désingularisation plongée d'une surface, la description des diviseurs exceptionnels, leurs types topologiques, les multiplicités et les relations d'incidence est d'une complexité comparable aux désingularisations en dimension trois. Ces descriptions sont nécessaires pour certaines applications.

Dans ce travail, on donne la description de quelques exemples simples, où la dimension de plongement (i.e. la dimension de l'espace tangent de Zariski) est minimum, donc des hypersurfaces.

Il existe plusieurs méthodes pour obtenir des désingularisations plongées d'hypersurfaces, mais les descriptions explicites sont rares.

Les résultats résumés ici sont une partie du contenu de plusieurs exposés réalisés aux Universités de Harvard et Northeastern en 1987 (dans un séminaire conjoint organisé par H. Hironaka, D. T. Lê, M. Spivakovsky et l'auteur) et à l'Institut Fourier en 1988.

Cette présentation introductive de quelques désingularisations plongées constitue une base expérimentale pour l'étude des liens avec les courbes tracées sur la surface et les points fixes des courbes polaires qui interviennent dans la transformation de Nash.

\section{Exemples et descriptions des désingularisations plongées.}

Les singularités de surfaces choisies sont quelques cas de points doubles rationnels. Ces exemples sont déjà suffisamment non triviaux pour illustrer quel- 
ques phénomènes généraux, d'où leur ubicuité. Ce sont des singularités absolument isolées, i.e. on peut les désingulariser en faisant une itération d'éclatements ponctuels, c'est-à-dire des éclatements de centre l'idéal maximal d'un point. On obtient ainsi des désingularisations qui sont minimales et qui, de plus, sont des bonnes désingularisations. En suivant les diviseurs exceptionnels qui apparaissent dans l'espace ambiant, on construit une désingularisation plongée en faisant des éclatements de courbes (ou de points) de tangence de ces diviseurs avec le transformé strict de la surface.

Soit $O$ l'origine de $C^{3}$; la notation choisie pour l'éclatement de $C^{3}$ de centre $O$ est standard: l'éclaté $\widehat{\boldsymbol{C}}^{3}$ est recouvert par les ouverts affines $U_{\imath}, 1 \leqq i \leqq 3$, où chaque $U_{\imath}$ est une copie de $C^{3}$ avec coordonnées $\left(X_{\imath}, Y_{\imath}, Z_{\imath}\right)$, de sorte que $U_{\imath}$ est l'ouvert où le $i$-ème générateur de l'idéal maximal $m=(X, Y, Z)$ de l'origine de $C^{3}$ devient le générateur de l'idéal principal $m \Theta_{\hat{c}^{3}}$ (i.e. $\left(X_{1}\right)$ pour $U_{1},\left(Y_{2}\right)$ pour $U_{2},\left(Z_{3}\right)$ pour $\left.U_{3}\right)$; autrement dit ce générateur donne une équation locale du diviseur exceptionnel $D$ de l'éclatement, diviseur qui est isomorphe à un plan projectif $\boldsymbol{P}^{2}$. On dénotera $\sigma$. un éclatement ponctuel. Un éclatement de centre une courbe est noté $\bar{\sigma}_{.}$. Si la courbe éclatée est rationnelle (lisse), le diviseur exceptionnel introduit par l'éclatement dans l'espace ambiant de dimension 3 est isomorphe à une surface réglée rationnelle: on dénotera $F_{m}$ une surface isomorphe à $\boldsymbol{P}_{\boldsymbol{P}_{1}}\left(\mathcal{O}_{\boldsymbol{P}_{1}} \oplus \mathcal{O}_{\boldsymbol{P}_{1}}(m)\right)$ avec $m \geqq 0$; en particulier on a $F_{0}=\boldsymbol{P}^{1} \times \boldsymbol{P}^{1}$. Si $m>0$, on identifiera la seule courbe dans $F_{m}$ avec auto-intersection négative: $-m$. Les diviseurs exceptionnels des désingularisations plongées, dans les exemples considérés, sont des surfaces rationnelles. On dénotera $R_{n}$ une surface isomorphe à $\boldsymbol{P}^{2}$ éclatée en $n$ points; on donnera aussi le graphe dual des courbes de la fibre exceptionnelle de la surface rationnelle considérée, graphe pondéré par les auto-intersections. Par exemple

$$
D \cong R_{3}\left(\ddot{\sigma}^{2}-\sigma^{-2}-1\right)
$$

dénote un diviseur $D$ isomorphe à $\boldsymbol{P}^{2}$ éclaté en 3 points, où le deuxième point éclaté appartient à la fibre exceptionnelle du premier éclatement et le troisième point éclaté appartient à la fibre exceptionnelle du deuxième éclatement mais pas à celle du premier. D'où le graphe dual pondéré, car l'éclatement d'un point $x$ dans une surface lisse fait baisser d'une unité l'auto-intersection du transformé strict $\hat{C}$ d'une courbe $C$ portant $x$, par rapport à l'auto-intersection de $C$. En général la donnée d'un tel graphe ne suffit pas pour déterminer l'ordre des éclatements, mais cette donnée suffit dans les exemples considérés. Une surface $F_{1}$ est isomorphe à $R_{1}$, c'est le seul cas commun entre les $F_{m}$ et les $R_{n}$.

Dans la description d'une désingularisation plongée $\sigma: \hat{V} \rightarrow V$ de la surface plongée $S \hookrightarrow V$, si $\sigma^{-1}(S)=\hat{S}+\sum m_{a} D_{a}$ est la décomposition, comme cycle, en composantes irréductibles du transformé total de $S$, l'intersection d'un diviseur exceptionnel $D_{a}$ avec le transformé strict $\hat{S}$ de $S$ est décomposée aussi en composantes irréductibles avec la notation $D_{a} \cap \hat{S}=\sum d_{a}^{(i)}$, et la courbe intersection de deux composantes $D_{a}, D_{b}$ du diviseur exceptionnel est noté $(a b)=D_{a} \cap D_{b}$. On donnera, pour chaque exemple, une représentation des intersections de toutes 
les composantes du [transformé total avec un diagramme d'incidence, où les courbes d'intersection sont représentées par des traits.

\section{1, Singularité $A_{2}$}

Soit $S \subset V=C^{3}$ la surface d'équation $f=X Y+Z^{3}=0$, et $\sigma_{1}: V_{1} \rightarrow V$ l'éclatement de $V$ de centre l'origine. Le transformé strict $\hat{S}$ de $S$ est lisse, et son intersection $\hat{S} \cap D_{1}$ avec le diviseur exceptionnel est formée de deux courbes rationnelles lisses, $d_{1}$ et $d_{1}^{\prime}$. On a donc le désingularisation minimale de $S$, mais ce n'est pas une désingularisation plongée, car $D_{1}$ est tangente à $\hat{S}$ en $d_{1} \cap d_{1}^{\prime}$ (point selle).

En effet, les restrictions de $\sigma_{1}^{*} f$ aux ouverts $U_{\imath}, 1 \leqq i \leqq 3$, sont:

$$
\sigma_{1}^{*} f:\left\{\begin{array}{l}
X_{1}^{2}\left(Y_{1}+Z_{1}^{3} X_{1}\right) \\
Y_{2}^{2}\left(X_{2}+Z_{2}^{3} Y_{2}\right) \\
\left.Z_{3}^{2}\left(X_{3} Y_{3}+Z_{3}\right) \quad \text { (point selle : origine de } U_{3}\right) .
\end{array}\right.
$$

Pour obtenir une désingularisation plongée on peut faire un éclatement de centre $d_{1}$ ou de centre $d_{1}^{\prime}$, ce qui obligerait à un choix non canonique. Pour éviter un tel choix, on peut faire un éclatement de centre l'origine de $U_{3}$, i.e. $d_{1} \cap d_{1}^{\prime}$. On dénote $\sigma_{2}: V_{2} \rightarrow V_{1}$ cet éclatement; pour simplifier les notations on enlève les indices aux coordonnées de $U_{3}=C^{3}$ et on reprend les mêmes notations que pour $\sigma_{1}$ pour décrire les ouverts de ce nouvel éclatement.

Par abus de notation, on dénote par les mêmes symboles les transformés stricts de $\hat{S}, D_{1}, d_{1}$ et $d_{1}^{\prime}$ dans $V_{2}$; et par $D_{2}$ le diviseur exceptionnel introduit par $\sigma_{2}$. Maintenant $D_{1}$ est tangent à $\hat{S}$ en deux points selles (correspondant aux directions de $d_{1}$ et $d_{1}^{\prime}$ ), or la courbe rationnelle $d_{2}=D_{2} \cap \hat{S}$ contient les deux points selles pour $D_{1}$ et coïncide avec l'intersection $D_{1} \cap D_{2}$.

En effet, si $f_{1}=Z^{2}(X Y+Z)$ est la restriction de $\sigma_{1}^{*} f$ à $U_{3}$, les restrictions de $\sigma_{2}^{*} f_{1}$ aux trois ouverts de l'éclaté de l'origine de $U_{3}$ sont

$$
\sigma_{2}^{*} f_{1}:\left\{\begin{array}{l}
X_{1}^{3} Z_{1}^{2}\left(X_{1} Y_{1}+Z_{1}\right) \\
Y_{2}^{3} Z_{2}^{2}\left(X_{2} Y_{2}+Z_{2}\right) \\
Z_{3}^{2}\left(X_{3} Y_{3} Z_{3}+1\right)
\end{array}\right.
$$

$X_{1}=0$ (resp. $Y_{2}=0$, resp. $Z_{3}=0$ ) est l'équation locale de $D_{2}$, $Z_{1}=0$ (resp. $Z_{2}=0$ ) celle de $D_{1}$, et les autres facteurs donnent celle de $\hat{S}$.

Les diviseurs $\hat{S}, D_{1}$ et $D_{2}$ de $V_{2}$ ne sont pas à croisements normaux le long de la courbe $d_{2}$. L'éclatement de $V_{2}$ de centre $d_{2}$ introduit un nouveau diviseur et donne, par composition avec $\sigma_{1} \circ \sigma_{2}$ une désingularisation plongée.

Dénotons $\bar{\sigma}_{2}: \bar{V}_{2} \rightarrow V_{2}$ l'éclatement de centre $d_{2}$; pour le calculer il suffit de considérer (par la symétrie des équations) la restriction $f_{2}=X^{3} Z^{2}(X Y+Z)$ de $\sigma_{2}^{*} f_{1}$ au premier ouvert (où on a enlevé les indices). L'idéal à éclater est celui 
de $d_{2}$ dans cet ouvert: $(X, Z)$. Avec une notation standard analogue à celle d'un éclatement d'un point, avec deux ouverts $\bar{U}_{1}, \bar{U}_{3}$, on a:

$$
\bar{\sigma}_{2}^{*} f_{2}:\left\{\begin{array}{l}
X_{1}^{6} Z_{1}^{2}\left(Y+Z_{1}\right) \\
X_{3}^{3} Z_{3}^{6}\left(X_{3} Y_{3}+1\right)
\end{array} \quad \text { où } \quad \begin{array}{l}
\left(X_{1}, Z_{1}\right) \mapsto\left(X_{1}, X_{1} Z_{1}\right) \\
\left(X_{3}, Z_{3}\right) \mapsto\left(X_{3} Z_{3}, Z_{3}\right)
\end{array}\right\}=(X, Z)
$$

où $X_{1}=0$ (resp. $Z_{3}=0$ ) est l'équation du nouveau diviseur exceptionnel $D_{2}, Z_{1}=0$ celle de $D_{1}, X_{3}=0$ celle de $D_{2}$, et les autres facteurs celle de $\hat{S}$ dans chaque ouvert.

Soit $\sigma=\sigma_{1} \circ \sigma_{2} \circ \bar{\sigma}_{2}: \hat{V}=\bar{V}_{2} \rightarrow V$. La désingularisation plongée de $S \subset V$ obtenue est décrite de la façon suivante. La décomposition du transformé total de $S$ dans $\hat{V}$, comme cycle divisoriel avec les multiplicités de ses composantes irréductibles, est:

$$
\sigma^{-1}(S)=\hat{S}+2 D_{1}+3 D_{2}+6 D_{\overline{2}}
$$

On a

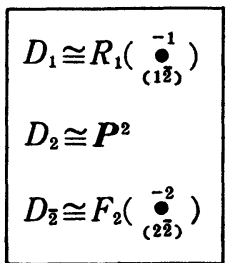

On a le diagramme d'incidence suivant (chaque trait représente une courbe intersection de deux diviseurs):

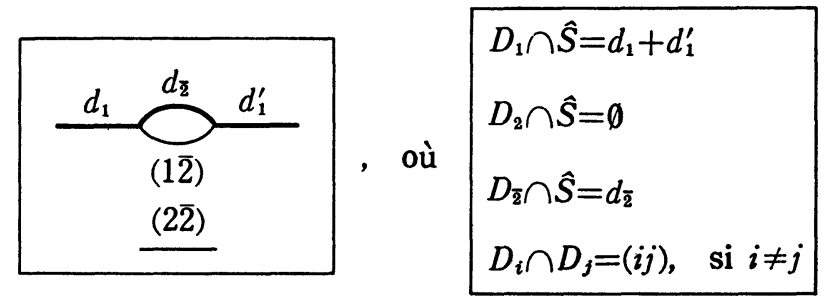

Plus généralement, pour une singularité de type $\boldsymbol{A}_{n}, n \geqq 1$, plongée dans son espace tangent de Zariski, la désingularisation minimale obtenue par une itération d'éclatements ponctuels est aussi une désingularisation plongée si $n$ est impair, et on a un point selle pour le point central du diagramme si $n$ est pair (d'où une désingularisation plongée analogue à celle de $\boldsymbol{A}_{2}$ pour la partie centrale du diagramme). 


\section{Rappels sur le cycle fondamental et la méthode de Tyurina.}

Soit $\sigma: \hat{S} \rightarrow S$ une désingularisation de $S$. On suppose que $O \in S$ est une singularité rationnelle, donc en particulier normale; c'est le cas des exemples ici considérés. Les singularités rationnelles sont absolument isolées, par conséquent l'idéal $\mathscr{M} \mathcal{S}_{\hat{S}}$ est localement principal, où $\mathscr{M}$ dénote l'idéal maximal en $O$. L'idéal $\mathscr{M O} \hat{s}$ définit un diviseur dans $\hat{S}$, diviseur qui coïncide avec le cycle fondamental. On peut calculer le cycle fondamental de la désingularisation avec la seule donnée de la matrice intersection. Soit $\sigma^{-1}(O)=d_{1} \cup \cdots \cup d_{r}$ la décomposition en composantes irréductibles de la fibre exceptionnelle de $\sigma$. Le cycle fondamental $\mathcal{Z}$ est l'unique cycle divisoriel minimal dans la famille des cycles divisoriels positifs, à support dans $\sigma^{-1}(O)$, tels qu'on ait $\left(\mathscr{L} \cdot d_{\imath}\right) \leqq 0, \forall i$, où $(\cdot)$ dénote la forme intersection du groupe de Picard de $\hat{S}$. La matrice intersection $\left(\left(d_{i} \cdot d_{j}\right)\right)$, $1 \leqq i \leqq r, 1 \leqq j \leqq r$ détermine $\mathcal{Z}$.

L'égalité $\mathscr{M} \mathcal{O}_{\hat{S}}=\mathcal{O}_{\hat{S}}(-\mathscr{Z})$ implique que l'on puisse déterminer l'ordre dans lequel on obtient les composantes irréductibles de la fibre exceptionnelle, dans la suite d'éclatements. En effet, les composantes $d_{\imath}$ telles qu'on ait $\left(\mathcal{Z} \cdot d_{\imath}\right)<0$ sont celles qui apparaissent dans le premier éclatement. Les composantes connexes (Tyurina) de la réunion des courbes $d_{\imath}$ telles qu'on ait $\left(\mathcal{L} \cdot d_{\imath}\right)=0$ sont les fibres exceptionnelles, dans $\hat{S}$, des singularités de l'éclaté de $S$ de centre $\mathscr{M}$; en itérant cette procédure pour les cycles fondamentaux des singularités des éclatés successifs, on détermine l'ordre d'apparition des autres composantes irréductibles. (réf. [A], [T]).

Dans les exemples suivants on donne les graphes duaux des désingularisations minimales (où chaque sommet représente une composante irréductible de la fibre exceptionnelle et chaque arête un point d'intersection des composantes correspondantes).

Des calculs combinatoires sur le cycle fondamental donnent l'ordre d'apparition des composantes irréductibles sur la surface éclatée et des calculs locaux suffisent alors pour compléter la description globale.

\section{Singularité de type $D_{4}$}

Soit $S \subset V=C^{3}$ la surface d'équation $X^{2}+Z\left(Y^{2}+Z^{2}\right)=0$. Le graphe dual de la désingularisation minimale est

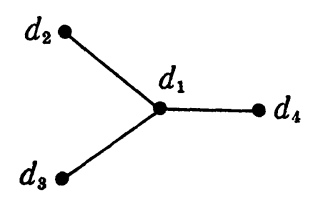

Le cycle fondamental est $\mathcal{Z}=2 d_{1}+d_{2}+d_{3}+d_{4}$; on a $\left(d_{i} \cdot d_{\imath}\right)=-2, \forall i$. Par suite $d_{1}$ est la courbe exceptionnelle du premier éclatement, la surface éclatée possède trois singularités du type $\boldsymbol{A}_{1}$, singularités qui donnent lieu aux com- 
posantes $d_{2}, d_{3}, d_{4}$. Soit $\sigma_{\imath}, 1 \leqq i \leqq 4$, les éclatements ponctuels correspondants; $D_{\imath}, 1 \leqq i \leqq 4$ les hypersurfaces exceptionnelles dans l'espace ambiant; $d_{\imath}=\hat{S} \cap D_{\imath}$, $1 \leqq i \leqq 4$, les courbes (rationnelles) intersection avec la transformée stricte $\hat{S}$ de $S ;(1 j)=D_{1} \cap D_{\jmath}, 2 \leqq j \leqq 4$. Le diagramme d'incidence de $\hat{S}$ et $D_{\imath}, 1 \leqq \imath \leqq 4$, est :

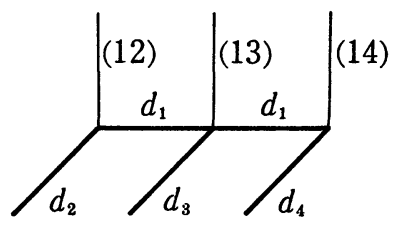

Le diviseur $D_{1}$ est tangent à $\hat{S}$ le long de $d_{1}$; pour obtenir des croisements normaux on fait l'éclatement $\bar{\sigma}_{1}$ de centre $d_{1}$, ce qui introduit un nouveau diviseur $D_{\overline{1}}$ isomorphe à $F_{2}$. Les transformées strictes de $D_{1}$ et $\hat{S}$ (notées avec les mêmes symboles) ont une intersection commune avec $D_{\overline{1}}$ le long d'une courbe rationnelle $d_{\overline{1}}$, donc ce n'est pas encore à croisements normaux. L'éclatement $\overline{\bar{\sigma}}_{1}$ de centre $d_{\overline{1}}$ introduit un nouveau diviseur $D_{\overline{1}}$ isomorphe à $F_{0}=\boldsymbol{P}_{1} \times \boldsymbol{P}_{1}$, et on obtient ainsi une désingularisation plongée, où on a $D_{1} \cap \hat{S}=\emptyset=D_{\overline{1}} \cap \hat{S}$. On note $d_{\overline{1}}$ l'intersection $D_{\overline{1}} \cap \hat{S}$, et $(a b)=D_{a} \cap D_{b}$, où $a$ et $b$ parcourent les indices des diviseurs, pour les intersections non vides.

Le diagramme d'incidence final est obtenu à partir du diagramme (2.2) en remplaçant les sous-diagrammes (2.3) par (2.4):
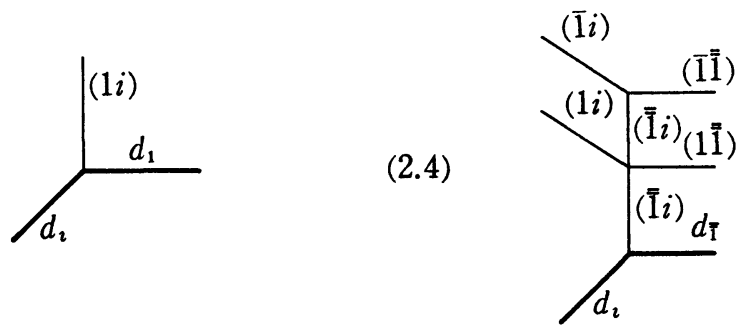

Soit $\sigma=\sigma_{1} \circ \sigma_{2} \circ \sigma_{3} \circ \sigma_{4} \circ \bar{\sigma}_{1} \circ \bar{\sigma}_{1}: \hat{V} \rightarrow V$; on a

$$
\sigma^{*}(S)=\hat{S}+2 D_{1}+3 D_{\overline{1}}+6 D_{\overline{1}}+4 D_{2}+4 D_{3}+4 D_{4} .
$$

Les diviseurs exceptionals sont des surfaces rationnelles du type suivant: 


$$
\begin{aligned}
& D_{1} \cong R_{3}\left(\begin{array}{ccc}
-1 & -1 & -1 \\
(12) & 0 & 0 \\
(13) & (14)
\end{array}\right) \\
& D_{\mathrm{I}} \cong F_{2}(\underset{\text { (if) }}{\stackrel{-2}{-2}}), \quad D_{\mathrm{1}} \cong F_{0}=\boldsymbol{P}^{1} \times \boldsymbol{P}^{1}
\end{aligned}
$$

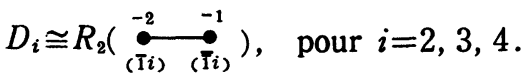

En effet, les restrictions des éclatements $\bar{\sigma}_{1}$ et $\overline{\bar{\sigma}}_{1}$ à chaque diviseur $D_{\imath}, 2 \leqq i \leqq 4$, sont des éclatements ponctuels au point de tangence des courbes $(1 i)$ et $d_{\imath}$, où l'ordre de contact est 2 .

Le diagramme d'incidence final est:

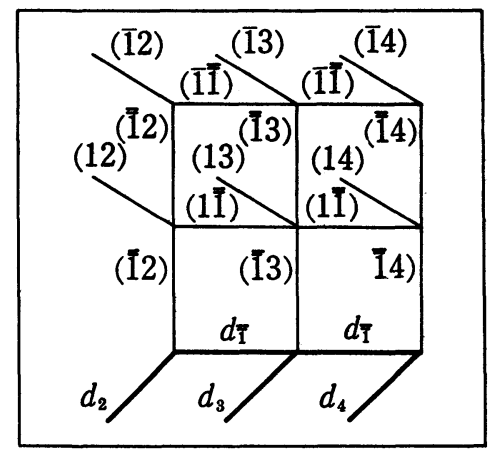

\section{Singularité $\boldsymbol{E}_{6}$}

Soit $S \subset V=C^{3}$ la surface d'équation $X^{2}+Y^{3}+Z^{4}=0$. Le graphe dual de la désingularisation minimale est

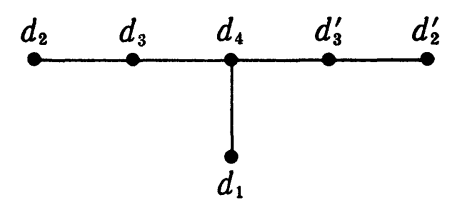

Le cycle fondamental est $\mathscr{L}=2 d_{1}+d_{2}+d_{2}^{\prime}+2 d_{3}+2 d_{3}^{\prime}+3 d_{4}$; on a $\left(d_{i} \cdot d_{\imath}\right)=-2$, $\forall i$. Par suite $d_{1}$ est la courbe exceptionnelle du premier éclatement $\sigma_{1}$, la surface éclatée possède une singularité de type $\boldsymbol{A}_{5} ; d_{2}$ et $d_{2}^{\prime}$ sont les composantes irréductibles de la courbe du deuxième éclatement $\sigma_{2}$, leur point d'intersection est une singularité de type $\boldsymbol{A}_{3} ; d_{3}$ et $d_{3}^{\prime}$ celles de $\sigma_{3}$, leur point d'intersection est une singularité de type $\boldsymbol{A}_{1}$; et $d_{4}$ celle de $\sigma_{4}$. Soit $D_{\imath}$ le diviseur exceptionnel, dans l'espace ambiant, introduit par $\sigma_{\imath}, 1 \leqq i \leqq 4$. 
Les diagrammes d'incidence successifs entre les diviseurs $D_{\imath}$ et le transformé strict $\hat{S}$ de la surface $S$ sont:

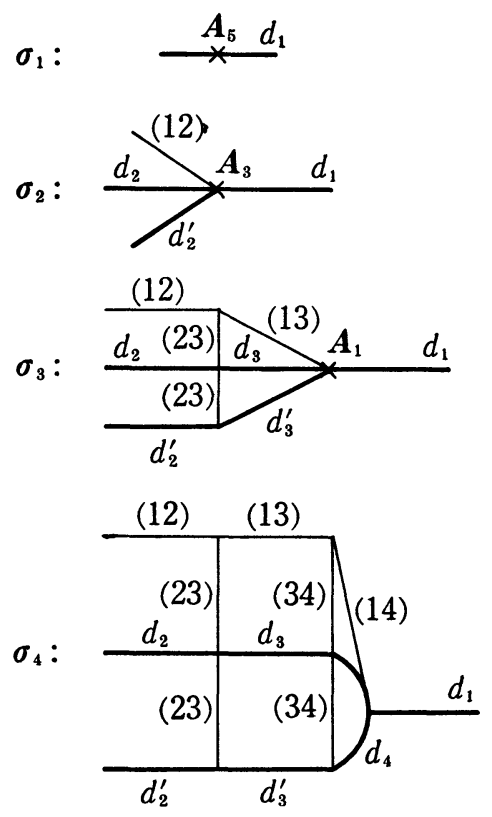

Le transformé total de $S$ par la composition des quatre éclatements se décompose comme cycle:

$$
\left(\sigma_{1} \circ \sigma_{2} \circ \sigma_{3} \circ \sigma_{4}\right)^{-1}(S)=\hat{S}+2 D_{1}+4 D_{2}+8 D_{3}+12 D_{4} .
$$

Or le diviseur $D_{1}$ est tangent à $\hat{S}$ le long de la courbe rationnelle $d_{1}$, donc la désingularisation n'est pas à croisements normaux. Dans la surface $D_{4}$, les courbes $d_{4}$ et (14) sont tangentes au point d'intersection avec $d_{1}$; l'ordre de contact est 2. Il faut faire deux éclatements de centre $d_{1}$, du même type que dans l'exemple précédent. Soit $\bar{\sigma}_{1}$ (resp. $\bar{\sigma}_{1}$ ) le premier (resp. le deuxième) éclatement, $D_{\overline{1}}$ (resp. $\left.D_{\overline{1}}\right)$ le diviseur exceptionnel correspondant.

La décomposition du cycle défini par le transformé total de $S$ par $\sigma=$ $\sigma_{1} \circ \sigma_{2} \circ \sigma_{3} \circ \sigma_{4}^{\circ} \bar{\sigma}_{1} \circ \bar{\sigma}_{1}$ est

$$
\sigma^{-1}(S)=\hat{S}+2 D_{1}+3 D_{\mathrm{I}}+6 D_{\mathrm{i}}+4 D_{2}+8 D_{3}+12 D_{4} \text {. }
$$

On a : 


$$
\begin{aligned}
& D_{1} \cong R_{3}\left(\begin{array}{ccc}
\stackrel{-2}{0} & -2 & -1 \\
(12) & (13) & (14)
\end{array}\right) \\
& D_{\overline{1}} \cong F_{2}\left(\underset{\left(\frac{\overline{1}}{1}\right)}{-2}\right), \quad D_{\overline{\mathrm{I}}}^{-2} \cong F_{0}=\boldsymbol{P}^{1} \times \boldsymbol{P}^{1}
\end{aligned}
$$

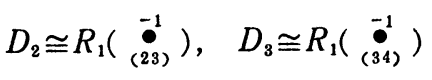

$$
\begin{aligned}
& D_{4} \cong R_{2}\left(\begin{array}{cc}
\stackrel{-2}{(14)} & \stackrel{-1}{(14)}
\end{array}\right) .
\end{aligned}
$$

Le diagramme d'incidence final est

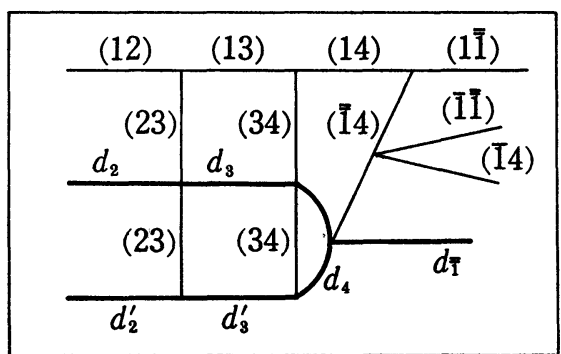

où $d_{a} \in \hat{S} \cap D_{a},(a b)=D_{a} \cap D_{b}$.

\section{Singularité de type $E_{8}$}

Soit $S \subset V=C^{3}$ la surface d'équation $X^{2}+Y^{3}+Z^{5}=0$. Le graphe dual de la désingularisation minimale est

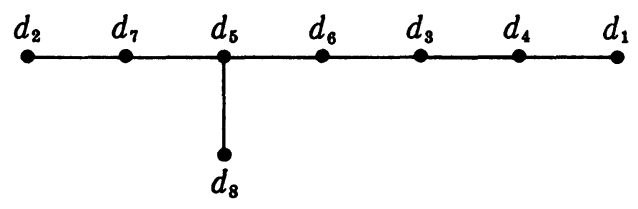

Le cycle fondamental est $\mathcal{Z}=2 d_{1}+2 d_{2}+4 d_{3}+3 d_{4}+6 d_{5}+5 d_{6}+4 d_{7}+3 d_{8}$; on a $\left(d_{i} \cdot d_{\imath}\right)=-2, \forall i$. Il faut huit éclatements ponctuels pour obtenir la désingularisation minimale; ils sont notés $\sigma_{\imath}, 1 \leqq i \leqq 8$. L'ordre total induit par l'ordre des indices choisi respecte l'ordre partiel dans lequel on fait les éclatements, i.e. si $\sigma_{\imath}$ précède $\sigma_{\jmath}$ alors $i<j$. Pour chaque $i, 1 \leqq i \leqq 8$, l'intersection $D_{i} \cap \hat{S}$ du diviseur dans l'espace ambiant $D_{\imath}$ avec le transformé strict $\hat{S}$ de la surface $S$ est une courbe irréductible $d_{\imath}$ (ce qui n'est le cas pour $\boldsymbol{A}_{n}(n>1)$ et $\boldsymbol{E}_{6}$. On peut construire le diagramme d'incidence, dans l'espace ambiant, de la composition 
des huit éclatements sans effectuer d'autres calculs que ceux des composantes de Tyurina successives.

En effet, l'irréductibilité de $D_{i} \cap \hat{S}, 1 \leqq i \leqq 8$ implique que si le diagramme d'incidence ensembliste de trois diviseurs est celui correspondant à la position général de trois hyperplans, alors le diagramme d'incidence de l'éclatement du point d'intersection des trois diviseurs est obtenu en remplaçant ce point par un triangle qui représente le nouveau diviseur exceptionnel:
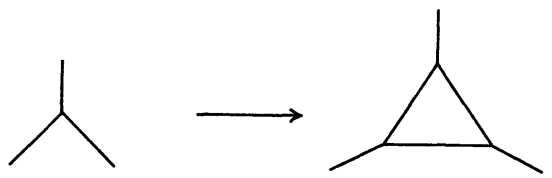

Le calcul des composantes de Tyurina donne les résultats suivants: soit $\hat{S}_{2}$ le transformé strict de $S$ par la composition d'éclatements $\sigma_{1} \circ \cdots \circ \sigma_{\imath-1} \circ \sigma_{\imath}$; alors $\hat{S}_{1}$ a une singularité de type $\boldsymbol{E}_{7} ; \hat{S}_{2}$ de type $\boldsymbol{D}_{6} ; \hat{S}_{3}$ deux singularités de type $\boldsymbol{A}_{1}$ et $\boldsymbol{D}_{4} ; \widehat{S}_{4}$ de type $\boldsymbol{D}_{4} ; \widehat{S}_{5}$ trois singularités de type $\boldsymbol{A}_{1}$, qui sont désingularisées par $\sigma_{6}, \sigma_{7}$ et $\sigma_{8}$. Par suite on obtient le diagramme d'incidence suivant, pour la composition $\sigma_{1} \circ \cdots \circ \sigma_{8}$ :

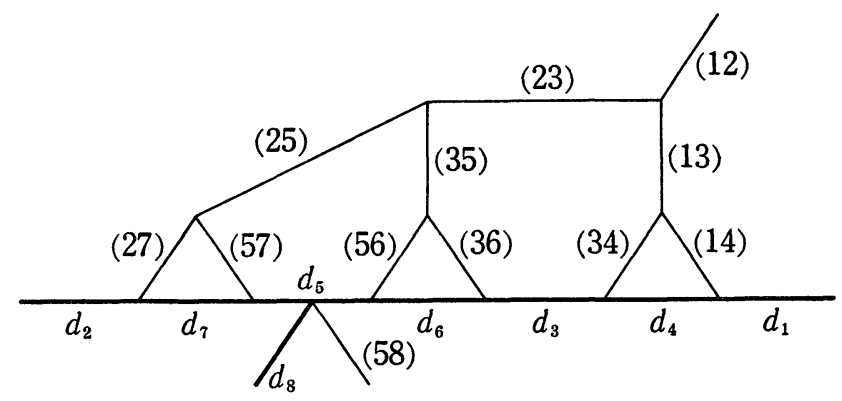

Un calcul local montre qu'il y a quatre diviseurs exceptionnels $D_{\imath}$ qui sont tangents à la transformée stricte $\hat{S}\left(=\hat{S}_{8}\right)$ le long des courbes (rationnelles) $d_{\imath}$, pour $i=1,2,3,5$. Par suite, dans les surfaces $D$, pour $j=4,6,7,8$, la courbe (ij) est tangente à $d_{\jmath}$, si $D_{i} \cap D_{\jmath} \neq \emptyset$. L'ordre de contact est deux, comme dans les exemples précédents. Pour obtenir une désingularisation plongée, il faut faire deux éclatements de centre $d_{\imath}$, pour $i=1,2,3,5$, éclatements notés $\bar{\sigma}_{\imath}$ et $\bar{\sigma}_{\imath}$; ces couples d'éclatements sont indépendants pour deux indices $i$ différents.

On obtient les multiplicités des diviseurs exceptionnels introduits par $\sigma_{\imath}$, $1 \leqq i \leqq 8$, à partir du graphe (4.1), de l'ordre déduit des cycles fondamentaux successifs, et des multiplicités des singularités intermédiaires. On a donc

$$
\left(\sigma_{1} \circ \cdots \circ \sigma_{8}\right)^{-1}(S)=\hat{S}+2 D_{1}+4 D_{2}+8 D_{3}+12 D_{4}+14 D_{5}+24 D_{6}+20 D_{7}+16 D_{8} .
$$

Soit $D_{\bar{\imath}}$ (resp. $D \overline{\bar{\imath}}$ ) le diviseur exceptionnel introduit par l'éclatement $\bar{\sigma}_{\imath}$ (resp. 
$\left.\overline{\bar{\sigma}}_{\imath}\right), i=1,2,3,5$. Soit $\sigma$ la composition des $\sigma_{\bar{i}} \circ \sigma_{\bar{\imath}}, i=1,2,3,5$, avec $\sigma_{1} \circ \cdots \circ \sigma_{8}$. Dans $\sigma^{-1}(S)$, la multiplicité de chaque $D_{i}$ est la même que celle qu'il a dans $\left(\sigma_{1} \circ \cdots \circ \sigma_{8}\right)^{-1}(S)$, car c'est le transformé strict. Soit $m_{\imath}$ la multiplicité de $D_{i}$, alors celle de $D_{\bar{i}}$ (resp. de $D_{\bar{\imath}}$ ), dans $\sigma^{-1}(S)$, est $m_{i}+1$ (resp. $\left.2 m_{i}+2\right)$. On a donc

$$
\begin{array}{rlll}
\sigma^{-1}(S)=\hat{S} & +2 D_{1}+4 D_{2} & +8 D_{3}+12 D_{4} & +14 D_{5}+24 D_{6}+20 D_{7}+16 D_{8} \\
+3 D_{\overline{\mathrm{1}}}+5 D_{\overline{\mathbf{2}}} & +9 D_{\overline{3}} & +15 D_{\overline{5}} \\
+6 D_{\overline{\mathrm{i}}}+10 D_{\overline{\mathrm{z}}} & +18 D_{\overline{\mathrm{z}}} & +30 D_{\overline{\mathrm{5}}}
\end{array}
$$

Les diviseurs exceptionnels sont des surfaces rationnelles du type suivant:

$$
\begin{aligned}
& D_{1} \cong R_{3}\left(\begin{array}{ccc}
-2 & -2 & -1 \\
(12) & (13) & (14)
\end{array}\right) \\
& D_{2} \cong R_{3}\left(\begin{array}{ccc}
-2 & -2 & -1 \\
(23) & -25) & (27)
\end{array}\right) \\
& D_{3} \cong R_{3}\left(\begin{array}{ccc}
\stackrel{-1}{0} & \stackrel{-2}{0} & -1 \\
(34) & (35) & (36)
\end{array}\right) \\
& D_{4} \cong R_{4}\left(\begin{array}{cccc}
-2 & -1 & -1 & -1 \\
(\overline{1} 4) & (\bar{I} 4) & (\overline{3} 4) & (\overline{3} 4)
\end{array}\right) \\
& D_{5} \cong R_{3}\left(\begin{array}{ccc}
-1 & -1 & -1 \\
(56) & 0 & 0 \\
(57) & (58)
\end{array}\right)
\end{aligned}
$$

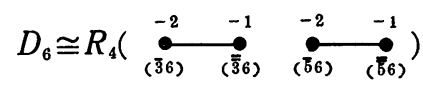

$$
\begin{aligned}
& D_{7} \cong R_{4}\left(\begin{array}{cccc}
\stackrel{-2}{-2} & -1 & -2 & -1 \\
(\overline{2} 7) & (\overline{\overline{2}} 7) & (\overline{5} 7) & (\overline{\overline{5}} 7)
\end{array}\right) \\
& D_{8} \cong R_{2}\left(\begin{array}{cc}
\stackrel{-2}{-2} & -1 \\
(\overline{5} 8) & \stackrel{(0)}{(58)}
\end{array}\right) \\
& D_{\bar{i}} \cong F_{2}(\underset{(\dot{i} \bar{i})}{-2}) \text { et } \\
& D_{\bar{i}} \cong F_{0}=\boldsymbol{P}^{1} \times \boldsymbol{P}^{1} \text { pour } i=1,2,3,5 \text {. }
\end{aligned}
$$

Le diagramme d'incidence de toutes les composantes irréductibles de $\sigma^{-1}(S)$ est obtenu à partir du diagramme (4.2), en remplaçant les sous-diagrammes de type (2.3) attachés à $d_{1}, d_{2}, d_{3}$ et $d_{5}$ par des sous-diagrammes du type (2.4). La partie centrale correspondante à $d_{5}$ est un diagramme de type (2.5). Le résultat final est le diagramme suivant: 


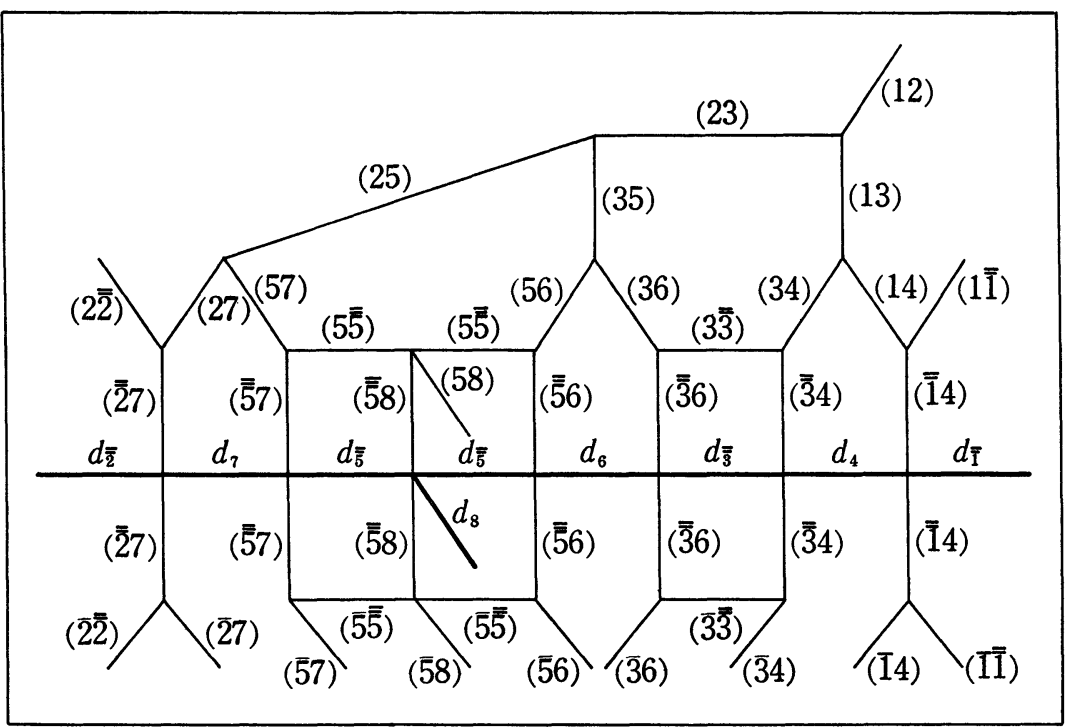

où $d_{a}=D_{a} \cap \hat{S},(a b)=D_{a} \cap D_{b}$.

Remarques.-Dans les exemples précédents, la désingularisation plongée obtenue induit la désingularisation minimale de la surface considérée, sauf dans le cas $\boldsymbol{A}_{n}, n$ pair. Dans ce dernier cas, il existe aussi des désingularisations plongées dont la désingularisation induite de la surface soit minimale, mais elles sont obtenues avec un choix non canonique.

Dans le cas général, il faudrait peut-être considérer comme point de départ une désingularisation minimale à croisements normaux où l'idéal maximal devient inversible, et remplacer l'étude du cycle fondamental par celle du cycle maximal.

\section{RÉFÉRENCES}

[A] Artin, M., On isolated rational singularities of surfaces, Amer. J. Math., 88 (1966), 129-136.

[A-H-V] Aroca, J.M., Hironaka, H. And Vicente, J.L., Desingularization theorems, Mem. de Mat. 30, Madrid, 1977.

[T] Tyurina, G.N., Absolute isolatedness of rational singularities, Func. Anal. Appl., 2 (1968), 324-332.

INSTITUT FOURIER

Laboratoire de Mathematiques associé au C.N.R.S.

Université de GRENOBLE I

B.P. 74

38402 st Martin D'hères Cedex

(FRANCE) 